\title{
Table of cases and practice directions
}

Abrath v North Eastern Railway Co [1886] 11 App Cas 247 ............................. 101

Airedale NHS Trust $v$ Bland [1993] AC 789 ......................................... 136, 137

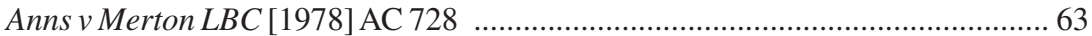

Armagas Ltd v Mundogas SA (The Ocean Frost) [1985] 1 Ll Rep 1 ................... 129

Att-Gen v Blake [2001] 1 AC 268 ................................................... 75, 79, 118

Att-Gen's Reference (No 2 of 1999) [2000] QB 796 ......................................... 101

Bank of Credit and Commerce International SA v Ali [2002] 1 AC 251 ................. 45

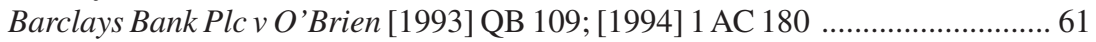

Beswick v Beswick [1966] Ch 538 (CA); [1968] AC 58 .............................. 29, 67

Birmingham CC v Oakley [2001] 1 AC 617 ...................................... 11, 42, 162

Black-Clawson Ltd v Papierwerke Waldhof-Aschaffenburg AG [1975] AC 591 .... 47

Blackpool \& Fylde Aero Club Ltd v Blackpool BC [1990] 1 WLR 1195 ...... 114, 157

Bolton (HL) (Engineering) Co Ltd v TJ Graham \& Sons Ltd [1957] 1 QB 159 ...... 97

Bonham's case (1610) 8 Co Rep 114a ..................................................... 31-32

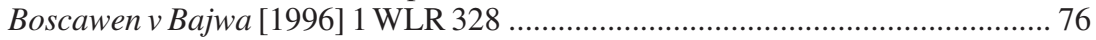

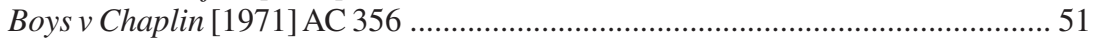

Bradford Corporation v Pickles [1895] AC 587 ............................................... 59

British Airways Board v Laker Airways Ltd [1985] AC 58 ................................. 74

Broome v Cassell \& Co Ltd [1972] AC 1027 ......................................................... 81

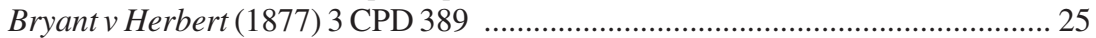

Burris v Azadani [1995] 1 WLR 1372 ....................................................... 68, 78

Caparo Industries plc v Dickman [1990] 2 AC 605 ............................................... 50

Chartbrook Ltd v Persimmon Homes Ltd [2009] 1 AC 1101 ................................ 45

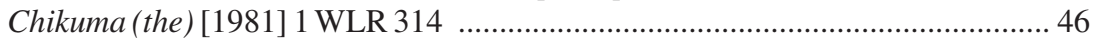

Citizens'Life Assurance Co Ltd v Brown [1904] AC 423 ................................... 101

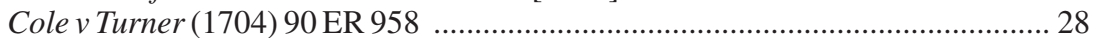

Co-operative Insurance Society Ltd v Argyll Stores Ltd [1998] AC 1 .................... 78

Council of Law Reporting v Att-Gen [1972] Ch 73 ............................................. 30

Courtney \& Fairbairn v Tolani Brother (Hotels) Ltd [1975] 1 WLR 297 ............ 157

Cozens v Brutus [1973] AC 854 ............................................................................. 44

Crawford Adjusters (Cayman) Ltd v Sagicor General Insurance (Cayman) Ltd

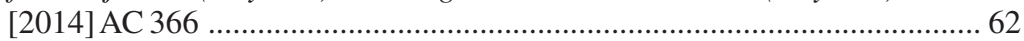

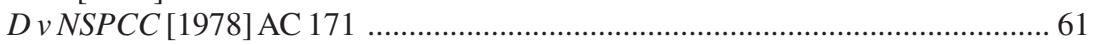

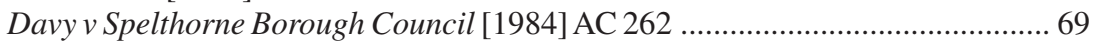

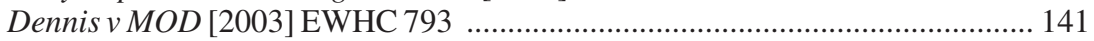

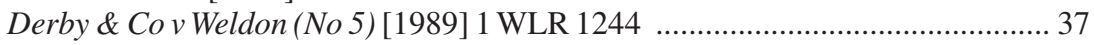

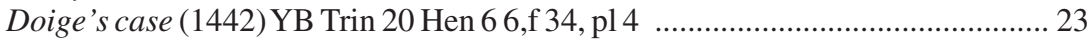

Donoghue v Stevenson [1932] AC 562 ................................................... 51, 52

DPP v Kent and Sussex Contractors Ltd [1944] KB 146 ................................. 101 
Dunne v NW Gas Board [1964] 2 QB 806

Elguzouli-Dafv Commissioner of Police of the Metropolis [1995] QB 335 ..... 62, 63

Esso Petroleum Ltd v Southport Corporation [1953] 3 WLR 773 (QBD); [1954] 2

QB 182 (CA); [1956] AC 218 (HL) $71,73,78,90,102,161$

F(In re $)$ [1990] 2 AC 1 .................................................................... 136, 137

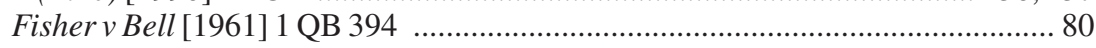

Foster $v$ Wheeler (1887) 36 Ch D 695 ............................................................... 29

Fothergill v Monarch Airlines Ltd [9181] AC 251 ............................................... 46

Goodwill v British Pregnancy Advisory Service [1996] 1 WLR 1397 ................... 78

Grant v Australian Knitting Mills Ltd [1936] AC 85 ........................................... 51

Hall (Inspector of Taxes) v Lorimer [1992] 1 WLR 939 ...................................... 95

Hannah Blumenthal (the) see Paal Wilson \& Co A/S v Partenreederei Hannah Blumenthal

Heaven $v$ Pender (1883) 11 QBD 503

Hedley Byrne \& Co v Heller \& Partners Ltd [1964] AC 465 ............... 122, 124, 155

Henderson v Merrett Syndicates Ltd [1995] 2 AC 145 ........................................ 27

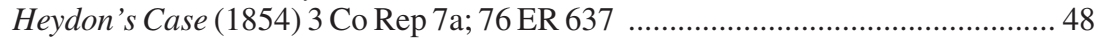

Hollywood Silver Fox Farm v Emmett [1936] 2 KB 468 .................................... 59

Home Office v Dorset Yacht Co [1970] AC 1004 .............................................. 110

Hoogendijk $v$ The Netherlands (2005) 40 EHRR SE 189 ................................... 85

Hunter v Canary Wharf Ltd [1997] AC 655 .......................................... 80, 113

Investors Compensation Scheme Ltd v West Bromwich Building Society [1998] 1 WLR 896 45,145

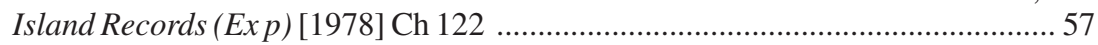

Jameel (Mohammed) v Wall Street Journal Europe Sprl [2007] 1 AC 359 ... 104, 105

Jetiva SA v Bilta (UK) Ltd [2015] UKSC 23 ..................................... 64, 98, 100

Jones $v$ Berkshire Area Health Authority (unreported) 2 July 1986 .................... 118

Jumbo King Ltd v Faithful Properties Ltd (1999) 2 HKCFAR 279 ........................ 45

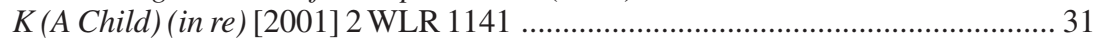

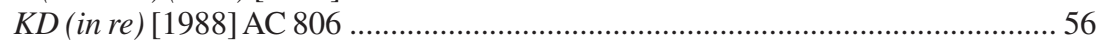

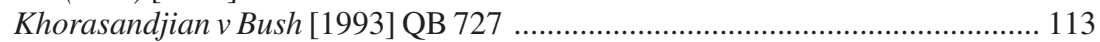

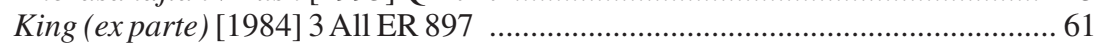

Kirin-Amgen Inc v Hoechst Marion Roussel Ltd [2005] 1 All ER 667 ................... 45

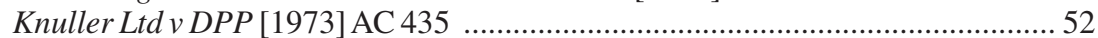

Lancashire County Council v Municipal Mutual Insurance Ltd [1997] QB 897 . 102

Les Laboratoires Servier v Apotex Inc [2014] UKSC 55; [2014] 3 WLR 1257 ...... 64

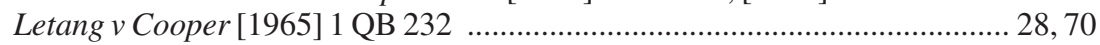

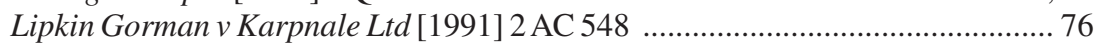

Lister v Hesley Hall Ltd [2002] 1 AC 215 ............................................ 101, 147

London, Chatham \& Dover Railway Co v S.E. Railway Co [1892] 1 Ch 120 ........ 75

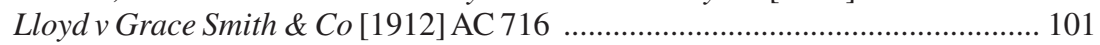

Lloyds Bank Ltd v Bundy [1975] QB 326 ........................................................ 112

Lupton v FA \& AB Ltd [1972] AC 634 ......................................................... 37, 51

Mackintosh v Great Western Railway Co 4 Giff 683 .......................................... 75

Mannai Investment Co v Eagle Star Life Assurance [1997] AC 749 ..................... 144

Mansfield v Weetabix Ltd [1998] 1 WLR 1263 ................................................ 140

McFarlane v Tayside Health Board [2000] 2 AC 59 ..... 118, 119, 120, 122, 126, 127

McKay v Essex Health Authority [1982] QB 1166 ........................................ 119 
Mercedes Benz AG v Leiduck [1996] 1 AC 284

Meridian Global Funds Management Asia Ltd v Securities Commission [1995] 2

AC 500

Michael v Chief Constable of South Wales Police [2015] UKSC 2

Millerv Jackson [1977] QB 966 63,145

Milton Keynes BC v Nulty [2013] 1 WLR 1183 $68,89,94,108,159$

M'Intosh v Great Western Railway Co see Mackintosh v Great Western Railway

Mirams (In re) [1891] 1 QB 594 121

Mirvahedy $v$ Henley [2003] 2 AC 491 92,162

Mitchell (George) (Chesterfield) Ltd v Finney Lock Seeds [1983] QB 284 .......... 146

Moore v I Bresler Ltd [1944] 2 All ER 515 ...................................................... 101

Morris v C W Martin \& Sons Ltd [1966] 1 QB 716 ................................... 99, 100

Motherwell v Motherwell (1976) 73 DLR (3d) 62 .......................................... 113

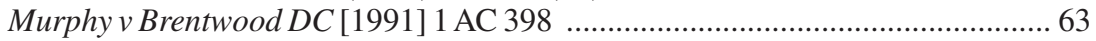

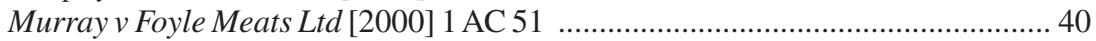

Myton v Woods (1980) WL 149543 ............................................................... 106

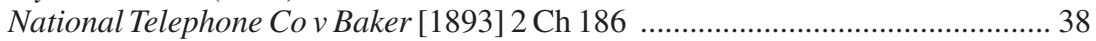

Norwich Pharmacal Co v Customs and Excise Commissioners [1974] AC 133 .... 74

Paal Wilson \& Co A/S v Partenreederei Hannah Blumenthal [1983] 1 AC 854;

[1982] 3 WLR 1149 (HL); [1982] 3 WLR 49 (CA) ................................... 51

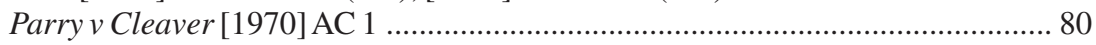

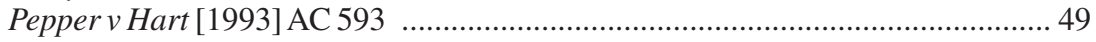

Photo Production Ltd v Securicor [1980] AC 827 ............................................. 99

Platform Funding Ltd v Bank of Scotland [2009] QB 426 ................................ 125

Prenn v Simmonds [1971] 1 WLR 1381 ...................................................... 145

Printing and Numerical Registering Co. v Sampson (1875) LR 19 Eq 462 ......... 146

Public Health Trust v Brown (1980) 388 So 1084 ............................................ 121

$R v$ East Sussex County Council [2015] UKSC 7 ................................................ 41

$R v$ Secretary of State for the Environment, Transport and the Regions, Exp Spath Holme Ltd [2001] 2 AC 349 .................................................................... 47

$R v$ Secretary of State for Work and Pensions [2015] UKSC 16 ........................... 85

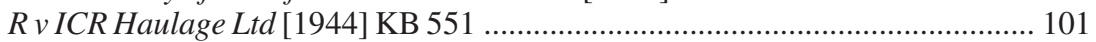

$R$ (Kadhim) v Brent Housing Board [2001] QB 955 ........................................... 50

$R$ (Quintavalle) $v$ Secretary of State for Health [2004] 2 AC 687 ......................... 32

Read v J Lyons \& Co [1945] 1 KB 216 (CA); [1947] AC 156 ..................... 117, 140

Rees v Darlington Memorial Hospital NHS Trust [2004] 1 AC 309 ............ 126, 127

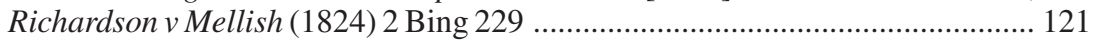

Ricket v Metropolitan Railway Co (1865) 5 B\&S 149 ............................................ 87

River Wear Commissioners v Adamson (1877) 2 App Cas 743 ............................ 32

Rowland $(R e)$ [1963] Ch 1 .............................................................. 93, 94, 159

Roxborough v Rothmans of Pall Mall (Australia) Ltd (2001) 208 CLR 516 ........ 114

Ruxley Electronics Ltd v Forsyth [1996] 1 AC 344 ....................................... 59, 90

Rylands v Fletcher (1866) LR 1 Ex 265 (Ex); (1868) LR 3 HL 330 (HL) ............... 38

$S$ (in re) [1995] 3 WLR 78 ........................................................................... 138

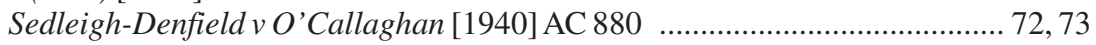

Shipton v Dog (see Doige's case)

Sienkiewicz v Greif(UK) Ltd [2011] 2 AC 229 ........................................ 129, 142

Spain (Kingdom of) v Christie, Mason \& Woods Ltd [1986] 1 WLR 1120 ............. 70 
Spartan Steel \& Alloys Ltdv Martin \& Co [1973] 1 QB 27 $60,96,157$

Spring v Guardian Assurance Plc [1995] 2 AC 296 124,161

Stone \& Rolls v Moore Stephens [2009] 1 AC 1391 102

Supply of Ready Mixed Concrete (No 2) (in re) [1995] 1 AC 456 ........................... 98

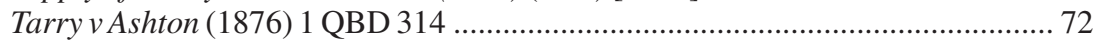

Tesco Stores Ltd v Brent LBC [1993] 1 WLR 1037 .............................................. 98

Tesco Supermarkets Ltd v Nattrass [1972] AC 153 ................. 97, 98, 99, 140, 160

Tinsley v Milligan [1994] 1 AC 340 64

Tomlinson v Congleton BC [2004] 1 AC 46 86

Transfield Shipping INC v Mercator Shipping INC [2009] 1 AC 61 132

Wainwright v Home Office [2004] 2 AC 406 89,90

Weaver $v$ Ward (1617) Hob 134; 80 ER 284 72

White v Chief Constable of South Yorkshire Police [1999] 2 AC 455 127,144

White v Jones [1995] 2 AC 207 79

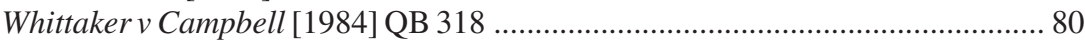

Wildtree Hotels Ltd v Harrow LBC [2001] 2 AC 1 ............................................ 87

Wilson v First County Trust Ltd (No 2) [2004] 1 AC 816 ...................................... 58

Woodland v Essex County Council see Woodland $v$ Swimming Teachers Association Woodland $v$ Swimming Teachers Association [2014] AC 537 ....... 50, 106, 107, 127, $144,159,162$

Young v Sun Alliance and London Insurance Ltd [1977] 1 WLR 104 43 